\title{
Influence of Turbulence on Naphthenic Acid Corrosion of SA210C
}

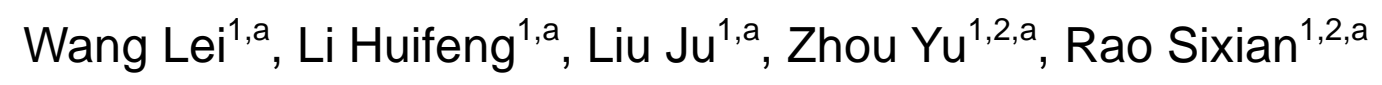

${ }^{1}$ School of mechanical engineering, Anhui university of technology,Ma'an'shan,243002,P R China

2.Hefei General Machinery Research Institute, National Technical Research Center on Safety

Engineering of Pressure Vessel and Pipelines,Hefei,230031,P R China

awlts1990@126.com

Keywords: naphthenic acid corrosion; low carbon steel; turbulence; high temperature corrosion

\begin{abstract}
The turbulence and flow rate around the specimens were analyzed by Fluent and results indicated that specimens installed in the pipeline varied the fluid flow direction, highly increased the flow rate nearby and formed strong turbulence around the specimens, the max turbulence in work areas could reach $10 \%$. Experiments about the influence of turbulence on corrosion rate showed that max influence of turbulence on average corrosion rate was $15.5 \%$ and obviously lower than the max influence of temperature;but in high turbulence areas the influence of turbulence on local corrosion depth was almost equal to the max influence of temperature. The corrosion depth on the specimens was consistent with the distribution of turbulence and the corrosion rate difference in work areas explained why ditches existed in high turbulence areas.
\end{abstract}

\section{Introduction}

Naphthenic Acid Corrosion(NAC) of refining equipment is still a question in the material science agenda. NAC occurs via chemical reaction with iron. Existing researches show that the main factors that could influence NAC are temperature, total acid number (TAN), type of naphthenic acid,content of active sulfide, flow rate and flow state of crude oil,type of materials ${ }^{[1]}$.

Temperature is an important factor that could significantly influence the rate of NAC, investigations show that NAC mainly occurs in the temperature range of $220 \mathrm{C}$ to $400 \mathrm{C}^{[2]}$. Total acid number(TAN) is also an important factor to evaluate NAC. Nugent and Dobis have reported that obvious NAC only occurs above critical TAN of $0.5 \mathrm{mg} \mathrm{KOH} / \mathrm{g}^{[3]}$. The corrosivity of naphthenic acid depends on the type of naphthenic acid, the corrosivity of various naphthenic acid differs obviously even under the same TAN ${ }^{4]}$.

The physical condition of fluid could also influence the corrosion rate. For example, NAC becomes very serious in the furnace tubes and transfer lines under the actions of high flow rate and two-phase flow. Gutzeit and Scattergood indicated that NAC was very serious at the dew-point temperature when the vapour condensated to liquid on the surface of metal ${ }^{[5-6]}$.

Flow rate and turbulence of crude oil could also obviously influence the corrosion rate of NAC. Under high temperature and high flow rate, low carbon steels would corrode quickly even under very low TAN level ${ }^{[7]}$.

Due to the complexity of NAC,it is impossible to correlate the corrosion rate of NAC with the influencing factors directly. The only way is to investigate the influence of the factors above individually. In this paper the influence of turbulence on NAC was discussed,the purpose was to uncover how and to what extent could it influence NAC.

\section{Experimental procedures}

Experiment conditions and devices. Because NAC would not occur below 220C and naphthenic acids cracked above 400C, the temperature selected in the experiments were 240, 280, 320 and 360C, the corrosion time was controlled at $8 \mathrm{~h}$. In order to investigate the influence of turbulence on NAC, two flushing angles $\left(0^{\circ}\right.$ and $\left.90^{\circ}\right)$ at pipe flow mode were selected to create turbulence in the experiments. The sketch of high temperature, high flow rate naphthenic acid 
corrosion simulation device was shown in Fig. $1^{[8]}$, the max flow rate in the device could reach $100 \mathrm{~m} / \mathrm{s}$ and the flow rate selected in the experiments was $20 \mathrm{~m} / \mathrm{s}$ at pipe flow mode and $80 \mathrm{~m} / \mathrm{s}$ at injection mode. the specimens were installed in the pipeline at pipe flow mode(Fig.2a) and the specimen was installed in the tray at injection mode(Fig.2b) .

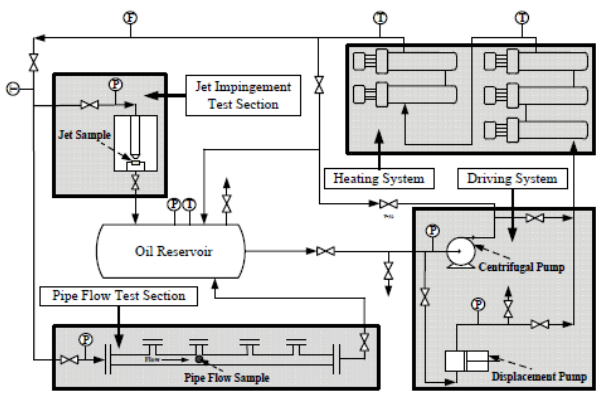

Fig.1 sketch of naphthenic acid corrosion simulation device

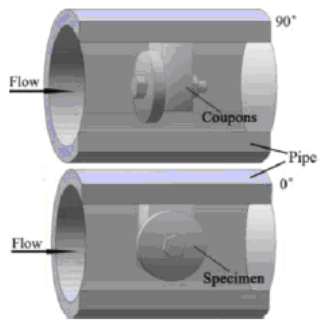

(a)

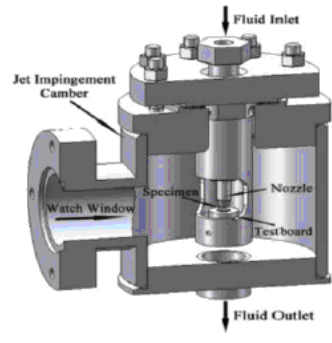

(b)

Fig.2 installation diagram of specimens (a- installation of specimens at pipe flow mode, b- installation of specimens at injection mode)

Experiment materials. ASME SA210C low carbon steel were selected as experiment materials. SA210C was quenched and then tempered, the compositions were listed in Table 1. The specimens were cut from sheets and machined to ring structure with external diameter of $18 \mathrm{~mm}$, internal diameter of $3 \mathrm{~mm}$. The specimens were ground to $1000 \#$ by abrasive papers and polished by $1 \mu \mathrm{m}$ polishing paste, and then the specimens were degreased and rinsed by de-ionized water. The weights of the specimens were recorded after drying for $12 \mathrm{~h}$. The medium selected in experiments was high temperature heat conductive oil (high-purity Dibenzyl toluene) mixed with refined naphthenic acids, the TAN was $4.6 \mathrm{mg} \mathrm{KOH} / \mathrm{g}$. The specimens were rinsed again by absolute ethyl alcohol and then degreased by acetone after experiments, subsequently cleaned by ultrasonic wave cleaner to remove corrosion products, rinsed again by de-ionized water and dried for 24h. Finally the weight loss of each specimen was measured by an analytical balance. According to Eq. (1), the average corrosion rate could be calculated by weight loss and exposed area.

Table.1 Chemical composition of test specimens (mass-\%)

\begin{tabular}{cccccccccc}
\hline Alloy & $\mathrm{C}$ & $\mathrm{Si}$ & $\mathrm{Mn}$ & $\mathrm{P}$ & $\mathrm{S}$ & $\mathrm{Cr}$ & $\mathrm{Cu}$ & $\mathrm{Mo}$ & $\mathrm{Ni}$ \\
\hline SA210C & 0.18 & 0.20 & 0.36 & $\leq 0.03$ & $\leq 0.030$ & - & $\leq 0.020$ & - & $\leq 0.025$ \\
\hline
\end{tabular}

Average Corrosion rate $\left(\mathrm{mm} \cdot \mathrm{a}^{-1}\right)=\frac{3650 \times(\text { Weight loss } / \mathrm{g})}{\text { Density of metal }\left(\mathrm{g} \cdot \mathrm{cm}^{-3}\right) \times \operatorname{Area}\left(\mathrm{cm}^{2}\right) \times \operatorname{time}(\text { day })}$

Analysis of turbulence and topography. The software of Fluent was used to analyze to the turbulence on the specimen.Three- dimensional digital microscope $\mathrm{KH}-7700$ was used to scan the corrosion topography of specimens.

\section{Experiment results and discussion}

Analysis of flow rate and turbulence around the specimens. The flow rate of medium in the pipeline was controlled at $20 \mathrm{~m} / \mathrm{s}$ at pipe flow mode and Fluent 6.2 was used to analyze the turbulence and flow rate around the specimens, the results were shown in Fig.3 and Fig.4. Fig.3 indicated that the max flow rate at flushing angle of $90^{\circ}$ could reach $50 \mathrm{~m} / \mathrm{s}$ around the specimen(Fig.3a), and the max flow rate at flushing angle of $0^{\circ}$ could reach $45 \mathrm{~m} / \mathrm{s}$ (Fig.3b). so the specimens installed in the pipeline varied the direction of fluid flow, highly increased the flow rate around and formed strong turbulence. Distributions of turbulence around the specimens were shown in Fig.4. at flushing angle of $90^{\circ}$ the highest turbulence could reach 10\%(Fig.4a); at flushing angle of $0^{\circ}$, the highest turbulence could reach $8 \%($ Fig.4b). the simulation results proved that very strong turbulence did exist around the specimens.

The greatest concern in the experiments was the turbulence on the work area. The turbulence 
distributions on the specimen were shown in Fig.5. The results indicated that the highest turbulence existed in the middle part on the specimen under flushing angle of $90^{\circ}$, while the highest turbulence existed in the area facing the fluid direction under flushing angle of $0^{\circ}$.Strong turbulence existed under two flushing angle and the highest turbulence both exceeded $8 \%$.

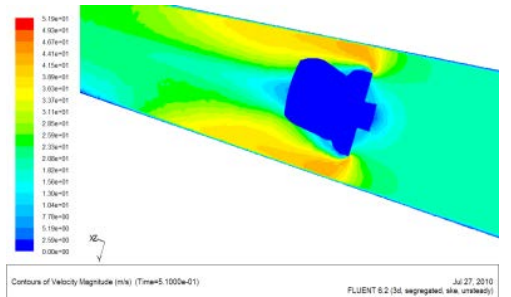

(a)

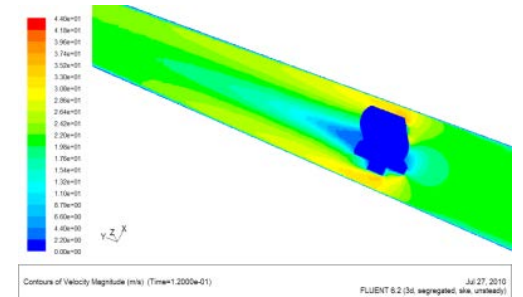

(b)

Fig.3 flow rate distribution around the specimens(a-flow rate distribution at $90^{\circ}$, b-flow rate distribution at $0^{\circ}$ )
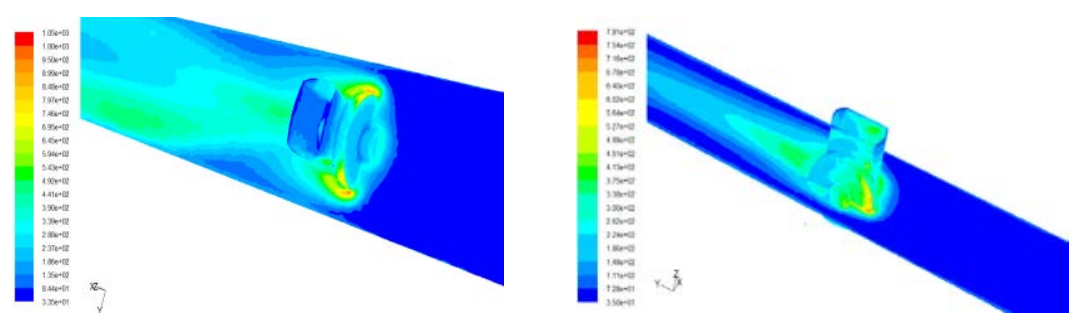

Fig.4 turbulence distribution around the specimens(a- turbulence at $90^{\circ}, \mathrm{b}$ - turbulence at $0^{\circ}$ )
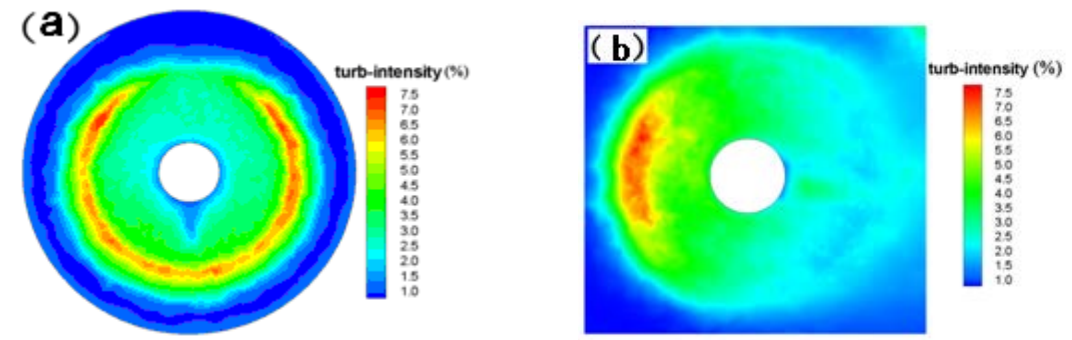

Fig.5 turbulence distribution on the work area of the specimens(a-turbulence at $90^{\circ}, \mathrm{b}$-turbulence at $0^{\circ}$ )

Correlation between turbulence and corrosion rate. Flow rate and turbulence were important factors that could influence corrosion rate of NAC, and severe corrosion frequently occurred on the spots where high flow rate and turbulence existed in the pipelines of crude oil refineries, but to what extent could turbulence influence the corrosion rate is still unknown. Experiments were carried out and the influence of turbulence on average corrosion rate, corrosion depth and local corrosion morphology were analyzed.

Average corrosion rates of SA210C at different temperatures and flushing angles were shown in Fig.6. It could be found that corrosion rates at $0^{\circ}$ and $90^{\circ}$ differed slightly at temperature of $240 \mathrm{C}$. The average corrosion rate at $0^{\circ}$ was $2.34 \mathrm{~mm} / \mathrm{a}$ and $2.53 \mathrm{~mm} / \mathrm{a}$ at $90^{\circ}$, the difference of corrosion rate was about $8.1 \%$. When temperature reached $280 \mathrm{C}$, the corrosion rate was $3.95 \mathrm{~mm} / \mathrm{a}$ at $0^{\circ}$ and $3.94 \mathrm{~mm} / \mathrm{a}$ at $90^{\circ}$,indicating that the corrosion rates increased with temperature and there was no obvious difference under two flushing angles. Corrosion rate increased more significantly when temperature reached $320 \mathrm{C}$, the corrosion rate was $6 \mathrm{~mm} / \mathrm{a}$ at $0^{\circ}$ and $6.9 \mathrm{~mm} / \mathrm{a}$ at $90^{\circ}$, and the difference was about $15 \%$. When temperature reached 360C, the corrosion rates decreased to $5.03 \mathrm{~mm} / \mathrm{a}$ at $0^{\circ}$ and $5.81 \mathrm{~mm} / \mathrm{a}$ at $90^{\circ}$, the corrosion rate difference was about $15.5 \%$. It is worthy of note that corrosion rate decreased obviously when temperature increased from 320 to 360C. As pointed out in Ref. [1], a maximum corrosion rate occurred among 270C to 320C when no active sulfide existed. Therefore the experimental results were consistent with the result in Ref. [1]. 


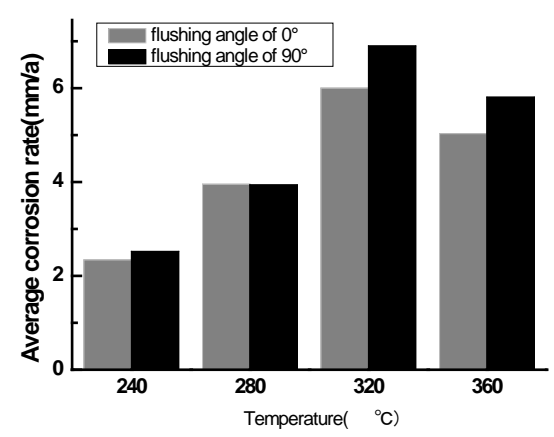

Fig.6 Average corrosion rate of SA210C under different temperature and flushing angle

It could be seen from Fig. 6 that the average corrosion rate of SA210C at 320C was 2.5 to 3 times of the corrosion rate at $240 \mathrm{C}$, but the highest corrosion rate difference under two flushing angle was $15.5 \%$. Temperature, compared to flushing angle and turbulence, was a more important factor for average corrosion rate. But the average corrosion rate could not accurately express the local influence of turbulence on NAC.

Analysis of local corrosion morphology of specimens(under temperature of 320C and flushing angle of $90^{\circ}$ ) showed that the corrosion depth differed obviously. In the low turbulence area the max corrosion depth was about $8.4 \mu \mathrm{m}$ (Fig.7a) and the average corrosion depth was $6.3 \mu \mathrm{m}$ calculated by the average corrosion rate $(6.9 \mathrm{~mm} / \mathrm{a})$, so the corrosion depth in low turbulence area were almost consistent with the average corrosion rate; But in the medium turbulence area the max corrosion depth was obviously higher than in the low turbulence area and the corrosion depth in Fig.7b could reach $17.5 \mu \mathrm{m}$; In the strong turbulence area(Fig.7c) the max corrosion depth could reach $21.2 \mu \mathrm{m}$ and even higher. The max corrosion depth in strong turbulence area was about 2.5 times of the corrosion depth in low turbulence area and the influence of strong turbulence in local spots was almost equal to the influence of temperature. So the influence of turbulence could not be ignored. Under other conditions similar conclusions could be obtained.

From the analysis above it could be confirmed that turbulence could significantly enhance the corrosion rate and the corrosion rate difference led to the formation of ditches in high turbulence areas. The ditch morphology is the standard feature of NAC in high turbulence areas and the experimental results explained why ditches existed in these areas.

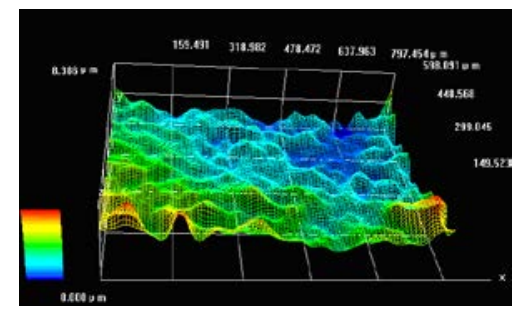

(a)

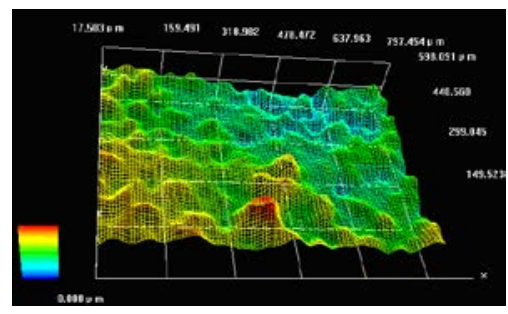

(b)

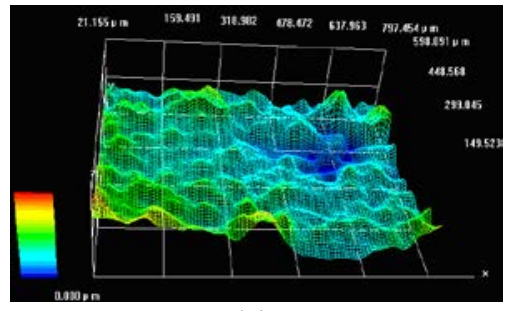

(c)

Fig.7 Corrosion depth and wireframe morphology in different turbulence area (a-low turbulence area,b-medium,c-strong turbulence area)

\section{Conclusion}

(1)Analog results indicated that specimens installed in the pipeline varied the fluid flow direction and highly increased the flow rate around and formed strong turbulence. The max turbulence in work areas could reach $10 \%$.

(2)Experimental results showed that turbulence could influence average corrosion rate of NAC, but the influence was obviously lower than the influence of temperature; but in local high turbulence areas the influence of turbulence on corrosion depth was very obvious and the extent was almost equal to the max influence of temperature.

(3)The corrosion rate difference in various turbulence areas led to the formation of ditches in 
high turbulence areas and the ditch morphology is the standard feature of NAC under high turbulence areas.

\section{Reference}

[1]Slavcheva E, Shone B, Turbull A. Review of naphthenic acid corrosion in oil refining[J].British Corrosion Journal,1999,34(2):125-131

[2]Groysman A, Brodsky N, Penner J,etc. Low Temperature Naphthenic Acid Corrosion Study [A],Corrosion 2007,Houston, NACE International,2007:569

[3]Nugent M J,Dobis J D. Experience with Naphthenic Acid Corrosion in low TAN Crudes[A]. Corrosion 98,Houston,NACE International,1998:577

[4]Groysman A,Brodsky N,Penner J,etc.Study of Corrosiveness of Acidic Crude Oil Corrosion and It’s Fractions[A],Corrosion2005,Houston,NACE International,2005:568

[5]Gutzeit J,Naphthenic Acid Corrosion in Oil Refineries[J],Materials Performance, 1997, 16(10):24-35

[6]Scattergood G L.Strong R C.Lindley W A,Naphthenic Acid Corrosion,An Update of Control Methods[A], Corrosion 87, NACE International,Houston,1987:197

[7]Xuedong Chen, Zhibin Ai, Tiecheng Yang,etc. Analysis Method of Failure Likelihood on Pressure Equipment with Combined Action of Multi-Failure Mechanism, PVP2010,Washington, 2010,25665

[8]Xu Peng,Chen Xuedong,Qin Zongchuan.etc,high-temperature and high-flow-rate testing device on naphthenic acid corrosion and test procedure[P].China,CN101477027,2009 